Address for Correspondence: Dr. Chikatoshi Katada, M.D., Ph.D.,Department of Gastroenterology, Kitasato University School of Medicine,

1-15-1 Kitasato, Minami-ku, Sagamihara, Kanagawa, 252-0374, Japan.

Email:ckatada@med.kitasato-u.ac.jp

\begin{tabular}{|l|}
\hline Access this article online \\
\hline Website: \\
www.intern-med.com \\
\hline DOI: \\
10.2478/jtim-2018-0007 \\
\hline Quick Response Code: \\
\hline \\
\\
\\
口) \\
\hline \\
\hline
\end{tabular}

\title{
Predisposing factors for chemotherapy- induced nephrotoxicity in patients with advanced esophageal cancer who received combination chemotherapy with docetaxel, cisplatin, and 5-fluorouracil
}

Junichi Mohri ${ }^{1}$, Chikatoshi Katada ${ }^{2}$, Marie Ueda ${ }^{3}$, Mitsuhiro Sugawara ${ }^{3}$, Keishi Yamashita ${ }^{4}$, Hiromitsu Moriya ${ }^{4}$, Shouko Komori ${ }^{5}$, Kazushige Hayakawa ${ }^{5}$, Wasaburo Koizumi ${ }^{2}$, Koichiro Atsuda ${ }^{1}$

${ }^{1}$ Research and Education Center for Clinical Pharmacy, Kitasato University School of Pharmacy, Tokyo, Japan; ${ }^{2}$ Department of Gastroenterology, Kitasato University School of Medicine, Kanagawa, Japan; ${ }^{3}$ Department of Pharmacy, Kitasato University Hospital, Kanagawa, Japan;

${ }^{4}$ Department of Surgery, Kitasato University School of Medicine, Kanagawa, Japan;

${ }^{5}$ Department of Radiology and Radiation Oncology, Kitasato University School of Medicine, Kanagawa, Japan

\section{ABSTRACT}

Background and Objectives: We retrospectively studied the predisposing factors for nephrotoxicity in the patients with advanced esophageal squamous-cell carcinoma who received combination chemotherapy with docetaxel, cisplatin, and 5-fluorouracil (DCF therapy). Methods: Between January 2010 and March 2014, 41 patients with Stage IB to III esophageal squamouscell carcinoma received the DCF therapy (docetaxel $70-75 \mathrm{mg} / \mathrm{m}^{2}$, day 1 ; cisplatin $70-75 \mathrm{mg} / \mathrm{m}^{2}$, day 1; 5-fluorouracil $750 \mathrm{mg} / \mathrm{m}^{2}$, days 1-5) in our hospital. Renal dysfunction was defined as a creatinine clearance (Ccr) of less than $60 \mathrm{~mL} / \mathrm{min}$. Predictors of nephrotoxicity were identified through logistic-regression analysis. Results: Nephrotoxicity developed in 20 patients and did not develop in 21 patients. Nephrotoxicity developed during the first course of DCF therapy in 16 patients, the second course in 3 patients, and the third course in 1 patient. The dose of DCF therapy was decreased in 8 patients with nephrotoxicity and 7 patients without nephrotoxicity. Multivariate analysis showed that a low Ccr level immediately before DCF therapy was an independent risk factor for the development of nephrotoxicity (odds ratio, 0.932; 95\% confidence interval, 0.876 to $0.992 ; P=0.027$ ). On receiver operating characteristic curve analysis, the optimal cutoff value of Ccr for the development of nephrotoxicity was $75.8 \mathrm{~mL} / \mathrm{min}$. The 2-year overall survival rate was $84.2 \%$ in patients with nephrotoxicity and $90.0 \%$ in patients without nephrotoxicity $(P=0.635)$. Conclusions: Low Ccr levels immediately before DCF therapy are a risk factor for the development of nephrotoxicity. Patients should therefore be carefully monitored.

Key words: esophageal cancer, docetaxel, cisplatin, 5-fluorouracil, chemotherapy-induced nephrotoxicity

\section{INTRODUCTION}

In Japan, a combination chemotherapy with docetaxel, cisplatin, and 5-fluorouracil (DCF therapy) is used to treat esophageal cancer, and good treatment outcomes have been obtained. ${ }^{[1-5]}$ However, cisplatin-induced nephrotoxicity is a serious clinical problem. ${ }^{[6-8]}$ Moreover, cases of renal impairment caused by docetaxel monotherapy have been reported. ${ }^{[9]}$ Predicting the risk of nephrotoxicity before treatment and taking countermeasures against such toxicity are considered beneficial for patients who receive DCF therapy.To our knowledge, however, studies assessing predisposing factors for DCF therapy-induced nephrotoxicity have yet to be reported. We therefore retrospectively studied the predisposing factors for nephrotoxicity in esophageal cancer patients who received the DCF therapy. 


\section{MATERIALS AND METHODS}

\section{Patients}

The study group comprised patients who were given a diagnosis of esophageal cancer in our hospital from January 1, 2010 through March 31, 2014 and met the following inclusion criteria: 1) a histologically confirmed diagnosis of squamous-cell carcinoma, 2) DCF therapy was received as initial chemotherapy, and 3) a diagnosis of clinical stage IB to III disease according to the seventh edition of the TNM classification by the Union for International Cancer Control. The exclusion criteria were as follows: 1) active double cancer in another organ, 2) a history of treatment for cancer in another organ, 3) use of a reduced dose of anticancer agents from the time of initial treatment, 4) concomitant treatment with radiotherapy, 5) nephrotoxicity (creatinine clearance $[\mathrm{Ccr}]<60 \mathrm{~mL} / \mathrm{min}$ ) or liver dysfunction (aspartate aminotransferase $>100 \mathrm{U} / \mathrm{L}$, alanine aminotransferase $>100 \mathrm{U} / \mathrm{L}$, total bilirubin of $>2.0 \mathrm{mg} / \mathrm{dL}$ ) at the onset of treatment,and 6) no laboratory data available for the period of several days before chemotherapy or during each course of treatment. The present study was approved by our institutional ethics committee.

\section{Chemotherapy}

DCF therapy for esophageal cancer consisted of docetaxel ( 70 to $75 \mathrm{mg} / \mathrm{m}^{2}$ ), given as a 1-hour, continuous intravenous infusion on day 1 ; cisplatin $\left(70\right.$ to $75 \mathrm{mg} / \mathrm{m}^{2}$ ), given as a 2 -hour, continuous intravenous infusion on day 1; and 5-fluorouracil $\left(750 \mathrm{mg} / \mathrm{m}^{2}\right)$, given as a continuous intravenous infusion on days 1 to 5 . Each course of chemotherapy was 21 days. In principle, patients received 3 courses of chemotherapy. To prevent nephrotoxicity, patients were given intravenous fluids for 6 days.

\section{Clinical variables}

The following clinical data were obtained from the medical records of the patients: age, sex, height, body weight, body-surface area, body-mass index, clinical disease stage, and disease history at the initiation of chemotherapy. In addition, the following variables were recorded at the start of each course of chemotherapy: performance status, serum albumin level, blood urea nitrogen (BUN) level, serum creatinine (Scr) level, Ccr, estimated glomerular filtration rate (eGFR), blood glucose level, hemoglobin A1c (HbA1c) level, systolic blood pressure, diastolic blood pressure, and concomitant medication. The urine volume on the day of chemotherapy and the total urine volume obtained in 5 days, starting on the day of chemotherapy were recorded. We also examined the administration method and dosage of each anticancer agent during each course of treatment and investigated the reason for any dose reduction. The clinical stage of cancer was classified according to the seventh edition of the TNM classification published by the Union for International Cancer Control. Performance status was evaluated according to the Eastern Cooperative Oncology Group classification. Ccr was estimated with the Cockcroft-Gault formula.

\section{Nephrotoxicity}

Serum creatinine levels are often used to evaluate renal function. However, Ccr and eGFR are more sensitive indicators of renal function and are therefore often used to adjust the dosage of anticancer agents. In our study, renal dysfunction was therefore defined as a Ccr of less than $60 \mathrm{~mL} / \mathrm{min}$, and the patients were classified into 2 groups: patients with nephrotoxicity and patients without nephrotoxicity. To accurately predict the development of nephrotoxicity, we analyzed the data for the course of treatment in which nephrotoxicity developed in patients with nephrotoxicity and the data during the final course of chemotherapy in patients without nephrotoxicity.

\section{Statistical analysis}

Univariate analysis was performed to determine explanatory variables. Categorical data were compared between the groups with the use of Fisher's exact tests and MannWhitney U tests. Quantitative data were compared with the use of Mann-Whitney $U$ tests. The significance level was set at $P<0.25$. Multicollinearity of variables for which significant differences were obtained was confirmed using the Spearman's rank correlation coefficient. For variables thatcorrelated, the variable with the smaller $P$ value obtained on intergroup comparison was used as an explanatory variable. The obtained explanatory variables were included in logistic regression analysis to identify the predictors of nephrotoxicity. The significance level was set at $P<0.05$. Receiver operating characteristic (ROC) curves were constructed to determine the cutoff values.

Overall survival was calculated from the date of starting the study treatment until the date of death or the final date of observation. Survival curves were estimated with the use of the Kaplan-Meier method. Survival curves were compared between the groups with the use of a log-rank test.The significance level was set at $P<0.05$. Statistical analysis was performed with the use of $\operatorname{IBM}^{\circledR}$ SPSS ${ }^{\circledR}$ Statistics software, Version 21.

\section{RESULTS}

Among 75 patients, 34 patients were excluded, and the other 41 patients were included in the analysis. The reasons for exclusion were active double cancers in other organs in 13 patients, a history of treatment for cancer in other organs in 5 patients, the concurrent use of radiotherapy in 1 patient, nephrotoxicity or liver dysfunction at the time of 
initial treatment in 12 patients, and no clinical laboratory data obtained in 3 patients. The patients included in the analysis were divided into 2 groups: 20 patients (49\%) who had nephrotoxicity (nephrotoxicity group) and 21 patients (51\%) with no nephrotoxicity (non-nephrotoxicity group). Nephrotoxicity developed during the first course of DCF therapy in 16 patients, the second course in 3 patients, and the third course in 1 patient. The doses of DCF therapy were reduced in 8 patients in the nephrotoxicity group and 7 patients in the non-nephrotoxicity group.

The results of univariate analysis of the predisposing factors for nephrotoxicity are show in Table 1 . A low $\operatorname{Ccr}(P$ $=0.010)$, high albumin level $(P=0.033)$, low urine volume (from the day of chemotherapy to day 5$)(P=0.124)$, low HbA1c level $(P=0.151)$, a high dose of cisplatin $(P=0.171)$ and high systolic blood pressure $(P=$ 0.210 ) were shown to be the potential predictors of the development of nephrotoxicity. Figure 1 shows the results of assessing multicollinearity among these 6 variables, evaluated with the use of Spearman's rank correlation coefficient. The serum albumin level significantly correlated with the dose of cisplatin, HbA1c level, and urine volume (from the day of chemotherapy to day 5) $(0.354, P=0.023$; $-0.742, P=0.014$; and $0.314, P=0.046$, respectively). On the basis of the results of univariate analysis and the assessment of multicollinearity, Ccr, albumin level, and systolic blood pressure were designated as explanatory variables, and multivariate analysis was performed (Table 2). Multivariate analysis showed that low Ccr immediately before chemotherapy was an independent risk factor for the development of nephrotoxicity (odds ratio, $0.932 ; 95 \%$ confidence interval, 0.876 to $0.992 ; P=0.027$ ).

To clarify the relation between the development of nephrotoxicity and the Ccrimmediately before chemotherapy, ROC curves were used to determine the cutoff values (Figure 2). The cut-off value of the Ccr level on ROC curve analysis was $75.8 \mathrm{~mL} / \mathrm{min}$.

To assess the relationship between the development of nephrotoxicity and treatment outcomes, overall survival

\begin{tabular}{|c|c|c|c|}
\hline & $\begin{array}{l}\text { Nephrotoxicity } \\
(n=20)\end{array}$ & $\begin{array}{l}\text { Non-nephrotoxicity } \\
(n=21)\end{array}$ & $P$ value \\
\hline Age (years) & $65(56-74)$ & $65(51-72)$ & 0.505 \\
\hline Gender (male/female) & $15 / 5$ & $18 / 3$ & 0.454 \\
\hline Body mass index $\left(\mathrm{kg} / \mathrm{m}^{2}\right)$ & $21.1(16.6-25.0)$ & $21.4(17.8-25.0)$ & 0.824 \\
\hline Clinical stage (IB/IIA/IIB/IIIA/IIIB/IIIC) ${ }^{*}$ & $2 / 6 / 5 / 2 / 2 / 3$ & $0 / 7 / 1 / 5 / 7 / 1$ & 0.335 \\
\hline Performance status $(0 / 1 / 2)$ & $10 / 9 / 1$ & $7 / 12 / 2$ & 0.266 \\
\hline Cisplatin dose $\left(\mathrm{mg} / \mathrm{m}^{2}\right)$ & $74.5(40.5-75.2)$ & $71.9(43.5-75.0)$ & 0.171 \\
\hline Concomitant nonsteroidal anti-inflammatory drugs & 2 & 4 & 0.663 \\
\hline Serum albumin level $(\mathrm{g} / \mathrm{dL})$ & $4.1(2.9-4.7)$ & $3.6(3.0-4.3)$ & 0.033 \\
\hline Blood urea nitrogen $(\mathrm{mg} / \mathrm{dL})$ & $13.2(7.0-26.7)$ & $13.4(6.4-23.2)$ & 0.938 \\
\hline Creatinine $(\mathrm{mg} / \mathrm{dL})$ & $0.76(0.39-0.99)$ & $0.72(0.46-0.90)$ & 0.948 \\
\hline Creatinine clearance $(\mathrm{mL} / \mathrm{min})$ & $74.5(63.0-106.4)$ & $83.1(65.6-109.1)$ & 0.010 \\
\hline Estimated glomerular filtration rate $\left(\mathrm{mL} / \mathrm{min} / 1.73 \mathrm{~m}^{2}\right)$ & $79.2(59.2-124.0)$ & $83.1(63.8-114.4)$ & 0.611 \\
\hline Blood glucose (mg/dL) & $111(87-169)$ & $116(90-181)$ & 0.374 \\
\hline Hemoglobin A1c (\%) & $5.6(5.3-6.3)$ & $6.4(5.5-7.1)$ & 0.151 \\
\hline Systolic blood pressure (mmHg) & $123(96-165)$ & $111(94-146)$ & 0.210 \\
\hline Diastolic blood pressure (mmHg) & $70(51-90)$ & $72(53-88)$ & 0.906 \\
\hline Urine volume for 1 day after chemotherapy $(\mathrm{mL})$ & $4292(2290-7136)$ & $4551(2713-7013)$ & 0.322 \\
\hline Urine volume for 5 days after chemotherapy $(\mathrm{mL})$ & $18587(14578-29558)$ & $22460(12851-29392)$ & 0.124 \\
\hline
\end{tabular}

*Union for International Cancer Control, $7^{\text {th }}$ edition. Values are presented as medians (ranges) except for gender, clinical stage, performance status, and concomitant nonsteroidal anti-inflammatory drugs. Statistical comparisons of categorical data between the groups were performed with Fisher's exact tests or Mann-Whitney U-tests. Statistical comparisons of quantitative data between the groups were performed with Mann-Whitney U-tests.

\begin{tabular}{lllll}
\hline Table 2: Risk factors for chemotherapy-induced nephrotoxicity - Multivariate analysis \\
\hline
\end{tabular}

RC: regression coefficient; $\mathrm{Cl}$ : confidence interval 


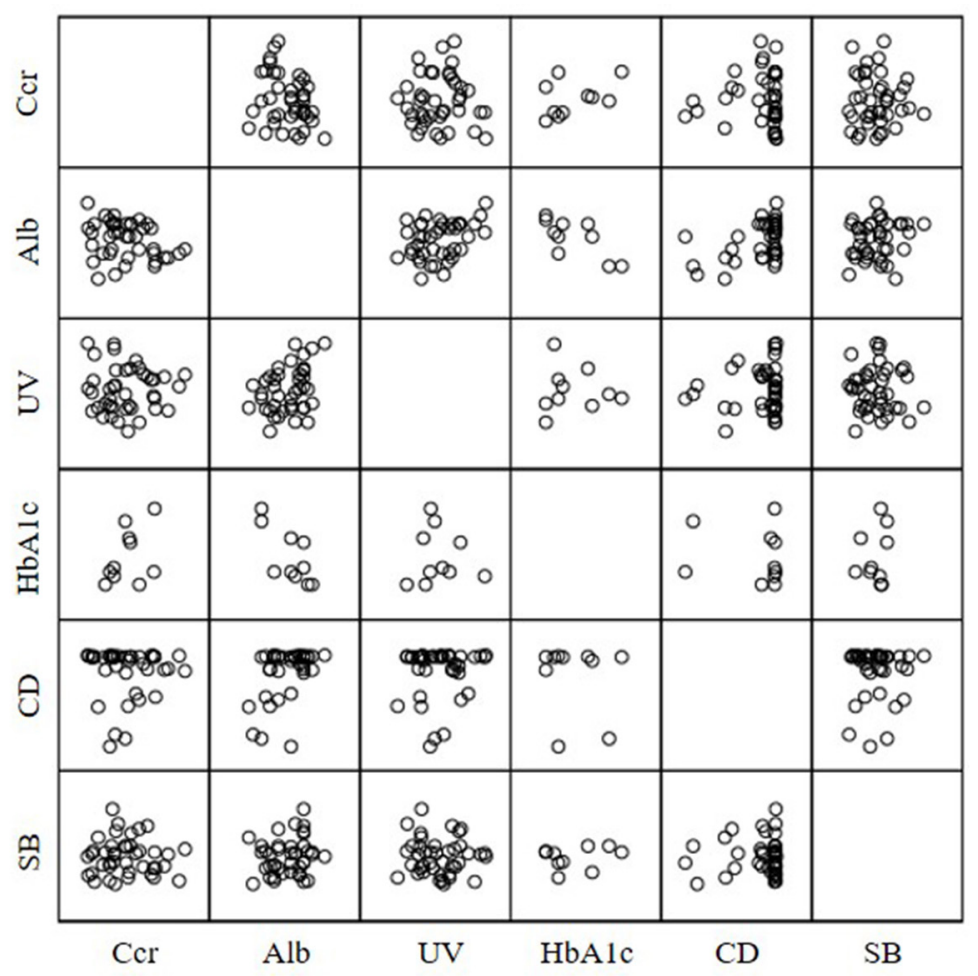

Figure 1: Correlations between predisposing factors on univariate analysis. Significant correlations (Spearman's rank correlation coefficient) were found between Alb levels and $\mathrm{CD}, \mathrm{HbA1c}$, and UV $(0.354, P=0.023 ;-0.742, P=0.014 ; 0.314, P=0.046)$. Ccr, Alb, UV, HbA1c, CD, and SB stand for creatinine clearance, albumin, urine volume for 5 days, hemoglobin A1c, cisplatin dose, and systolic blood pressure, respectively.

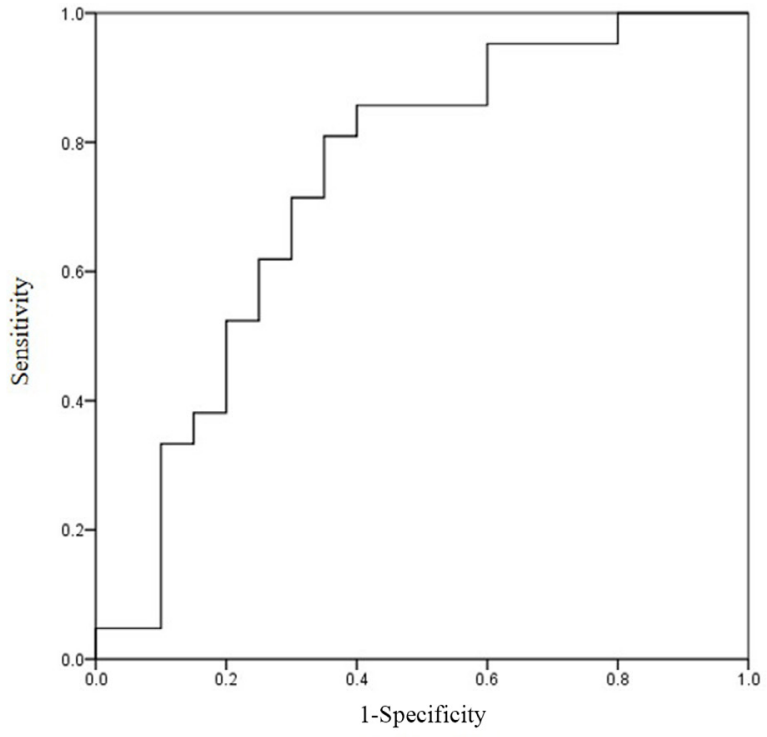

Figure 2: Receiver operating characteristic (ROC) curve of creatinine clearance for the development of nephrotoxicity. The cut-off value of creatinine clearance was $75.8 \mathrm{~mL} / \mathrm{min}$ (sensitivity, 0.81 ; specificity, 0.65 ). The area under the ROC curve was 0.736 .

was compared according to the presence or absence of nephrotoxicity (Figure 3). The median follow-up period was 36 months (range, 8 to 58 months) in patients with nephrotoxicity and 26 months (range, 4 to 60 months) in those without nephrotoxicity. The 2 -year survival rate was $84.2 \%$ in patients with nephrotoxicity and $90.0 \%$ in patients without nephrotoxicity $(P=0.635)$.

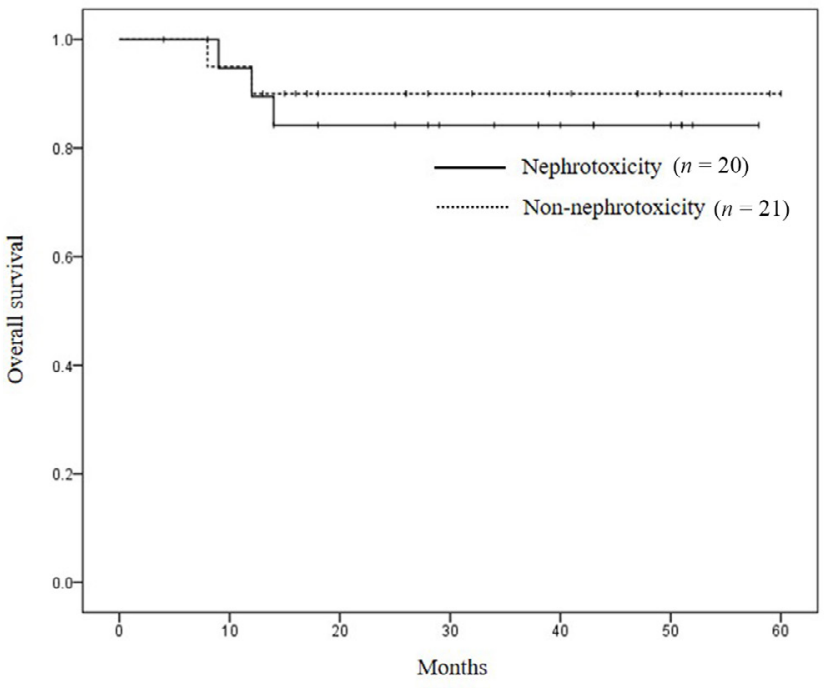

Figure 3: Kaplan-Meier overall survival curves according to the development or non-development of nephrotoxicity. There was no difference between the groups (log-rank test, $P=0.635$ ).

\section{DISCUSSION}

Our study showed that the Ccr value immediately before chemotherapy was a predictor of the development of nephrotoxicity in patients with esophageal cancer who received DCF therapy. The cutoff value of the Ccr was $75.8 \mathrm{~mL} / \mathrm{min}$, indicating that caution should be exercised 
for the development of DCF-induced nephrotoxicity in patients who have a Ccr of less than $75.8 \mathrm{~mL} / \mathrm{min}$ immediately before chemotherapy.Among the 17 patients who had a Ccr of less than $75.8 \mathrm{~mL} / \mathrm{min}$ immediately before chemotherapy in our study, 13 (76\%) had nephrotoxicity. Nephrotoxicity developed during the first course of DCF therapy in 9 patients, the second course in 3 patients, and the third course in 1 patient. Because many patients had nephrotoxicity during the first course of DCF therapy, patients should be carefully monitored.

The main mechanism causing cisplatin-induced nephrotoxicity is tubular impairment, which is dosedependent and cumulative. ${ }^{[6,10,11]}$ Recent attention has focused on biomarkers in urine as a sensitive index of nephrotoxicity. In particular, the measurement of renal tubular enzymes such as $\mathrm{N}$-acetyl- $\beta$-D-glucosaminidase (NAG); low-molecular-weight proteins in urine, such as $\alpha 1$ microglobulin, retinol-binding protein (RBP), and cystatin $\mathrm{C}$; and biomarkers in urine such as liver-type fatty acidbinding protein (L-FABP) has been found to be useful for evaluating renal tubular impairment. ${ }^{[12-14]}$ These biomarkers are more sensitive and specific than conventional markers of nephrotoxicity, such as Scr. On the basis of our results, these biomarkers were suggested to be useful for predicting the risk of nephrotoxicity soon after starting treatment in patients who have a Ccr level of less than $75.8 \mathrm{~mL} / \mathrm{min}$ immediately before chemotherapy.

In our study, the overall survival did not differ significantly between patients with nephrotoxicity and those without nephrotoxicity. However, there was a trend towards better overall survival in patients without nephrotoxicity. Therefore, predicting the risk and preventing the development of nephrotoxicity may contribute to an improvement in treatment outcomes.

Prophylactic treatment with magnesium preparations has been reported to provide protection against cisplatininduced nephrotoxicity. ${ }^{[8,15]}$ Because very few patients received the magnesium preparations in our study, the therapeutic usefulness could not be evaluated. Our results suggest that prophylactic treatment with magnesium preparations may contribute to a reduced incidence of nephrotoxicity in patients who have a Ccr of less than $75.8 \mathrm{~mL} / \mathrm{min}$ immediately before chemotherapy.

Kidera et al. reported that a performance status of 2 and regular treatment with nonsteroidal anti-inflammatory drugs (NSAIDs) are risk factors for nephrotoxicity induced by cisplatin-based regimens. ${ }^{[8]}$ Bhat $e t$ al. reported that severe acute renal injury can develop in patients who are severely malnourished. ${ }^{[16]}$ In our study, because few patients received regular treatment with NSAIDs or had a performance status of 2 or higher, we could not examine the relation between these factors and the development of nephrotoxicity. However, to establish methods for the prediction and prevention of nephrotoxicity associated with DCF therapy, the performance status, history of using NSAIDs, and nutritional status must be studied more extensively.

\section{CONCLUSION}

A low Ccr level immediately before DCF therapy is a risk factor for the development of nephrotoxicity. Patients must therefore be carefully monitored. Methods for the prediction and prevention of nephrotoxicity should be established in the future.

\section{ACKNOWLEDGEMENT}

The statistical methods of this study were reviewed by Dr. Mamoru Narukawa from Kitasato University School of Pharmacy.

\section{Conflicts of Interest}

The authors report no potential conflicts of interest relevant to this manuscript.

\section{REFERENCES}

1. Osaka Y, Shinohara M, Hoshino S, Ogata T, Takagi Y, Tsuchida A, et al. Phase II study of combined chemotherapy with docetaxel, CDDP and 5-FU for highly advanced esophageal cancer. Anticancer Res 2011; 31: 633-8.

2. Yokota T, Hatooka S, Ura T, Abe T, Takahari D, Shitara K, et al.Docetaxel plus 5-fluorouracil and cisplatin (DCF) induction chemotherapy for locally advanced borderline-resectable T4 esophageal cancer. Anticancer Res 2011; 31: 3535-41.

3. Tamura S, Imano M, Takiuchi H, Kobayashi K, Imamoto H, Miki $\mathrm{H}$, et al.; The Osaka Gastrointestinal Cancer Chemotherapy Study Group. Phase II study of docetaxel, cisplatin and 5-fluorouracil (DCF) for metastatic esophageal cancer (OGSG 0403). Anticancer Res 2012; 32: 1403-8.

4. Matsumoto A, Nishikawa K, Yuda M, Tanaka Y, Tanishima Y, Arakawa Y, et al. Early response of esophageal cancer to neoadjuvant chemotherapy with docetaxel-cisplatin-5-fluorouracil represents sensitivity: A Phase II study. Anticancer Res 2016; 36: 1937-42.

5. Katada N, Yamashita K, Katada C, Moriya H, Hosoda K, Mieno H, et al. Neoadjuvant chemotherapy using concurrent Docetaxel/CDDP/5-FU (DCF) in esophageal squamous cell carcinoma and its short-term prognosis. Esophagus 2014; 11: 173-81.

6. Arany I, Safirstein RL. Cisplatin nephrotoxicity. Semin Nephrol 2003; 23: $460-4$

7. Hartmann JT, Kollmannsberger C, Kanz L, Bokemeyer C. Platinum organ toxicity and possible prevention in patients with testicular cancer. Int J Cancer. 1999; 83: 866-9.

8. Kidera Y, Kawakami H, Sakiyama T, Okamoto K, Tanaka K, Takeda M, et al. Risk factors for cisplatin-induced nephrotoxicity and potential of magnesium supplementation for renal protection. PLoS ONE.2014; 9: e101902. 
9. Takimoto T, Nakabori T, Osa A, Morita S, Terada H, Oseto S, et al. Tubular nephrotoxicity induced by docetaxel in non-small-cell lung cancer patients. Int J ClinOncol 2012; 17: 395-8.

10. Madias NE, Harrington JT. Platinum nephrotoxicity. Am J Med 1978; 65: 307-14.

11. Miller RP, Tadagavadi RK, Ramesh G, Reeves WB. Mechanisms of cisplatin nephrotoxicity. Toxins 2010; 2: 2490-518.

12. Trof RJ, Maggio FD, Leemreis J, Groeneveld AB. Biomarkers of acute renal injury and renal failure. Shock 2006; 26: 245-53.

13. Kamijo A, Sugaya T, Hikawa A, Okada M, Okumura F, Yamanouchi $\mathrm{M}$, et al. Urinary excretion of fatty acid-binding protein reflects stress overload on the proximal tubules. Am J Pathol 2004; 165: 1243-55.

14. Obermüller N, Geiger H, Weipert C, Urbschat A. Current developments in early diagnosis of acute kidney injury. Int Urol Nephrol 2014; 46: 1-7.
15. Yoshida T, Niho S, Toda M, Goto K, Yoh K, Umemura S, et al. Protective effect of magnesium preloading on cisplatin-induced nephrotoxicity: A retrospective study. Jpn J Clin Oncol 2014; 44: 346-54.

16. Bhat ZY, Cadnapaphornchai P, Ginsburg K, Sivagnanam M, Chopra S, Treadway CK, et al. Understanding the risk factors and long-term consequences of cisplatin-associated acute kidney injury: An observational cohort study. PLoS ONE. 2015; 10: e0142225.

How to cite this article: Mohri J, Katada C, Ueda M, Sugawara $\mathrm{M}$, Yamashita $\mathrm{K}$, Moriya $\mathrm{H}$ et al. Predisposing factors for chemotherapyinduced nephrotoxicity in patients with advanced esophageal cancer who received combination chemotherapy with docetaxel, cisplatin, and 5-fluorouracil. J Transl Intern Med 2018; 6: $32-7$. 